\title{
Revisión y estado del arte de la corrosividad del etanol y sus mezclas con gasolina ${ }^{(*)}$
}

\author{
C. Berlanga*, M. V. Biezma** y J. Fernández*
}

\begin{abstract}
Resumen
Actualmente, el etanol se presenta como una fuente importante de combustible renovable para el sector de la automoción. Se sabe que los carburantes tradicionales, como la gasolina, no han causado problemas de corrosión, debido principalmente a su baja miscibilidad en el agua. En cambio, los biocarburantes en base alcohol pueden contener una considerable cantidad de agua y, además, se puede producir la oxidación parcial de sus componentes por su exposición a la atmósfera. Estas reacciones pueden ocasionar corrosión y posterior fallo, de cualquier componente metálico en contacto con las mezclas etanol-gasolina. En consecuencia, todos los sectores relacionados con la industria que emplean este combustible, empresas productoras, logística, dispensadores y fabricantes de vehículos, pueden estar afectados por esta problemática. Esto hace que este tema sea muy interesante, tanto desde un punto de vista científico como industrial. En este trabajo se revisa sistemáticamente todos los aspectos relacionados con la corrosión que produce el etanol y sus mezclas con gasolina, con el objetivo de conocer en profundidad el estado actual de las investigaciones, así como para orientar trabajos futuros que estén afectados, tanto de forma directa o indirecta con esta temática.
\end{abstract}

Palabras clave Etanol; Corrosión electroquímica; Corrosión bajo tensión; Inhibidores.

\section{Review and state-of-the-art of the corrosivity produced by ethanol and their mixtures with gasoline}

Abstract

Keywords

\begin{abstract}
Ethanol is currently an important source of renewable combustible for the automotive sector. It is known that traditional fuels such as gasoline have not caused corrosion problems due mainly to low miscibility with water. On the other hand, the alcohol based biofuels can contain a considerable amount of water, and in addition, partial oxidation of their components might be produced by exposition to the atmosphere, and this may lead to corrosion and subsequent failure of any metallic component in contact with the ethanol-gasoline blends. In consequence, all the industrial sectors related to this fuel, producing companies, logistics, dispensers and manufacturers of vehicles might be affected by this problem. This makes this topic very interesting, both from a scientific and an industrial point of view. This paper systematically reviews all aspects related to the corrosive behaviour of ethanol and ethanol-gasoline blends, with the aim of assessing the current state-of the-art as well as serving as a guide for further works that take the study of this subject matter.
\end{abstract}

Ethanol; Electrochemical corrosion; Stress corrosion cracking; Inhibitors.

\section{INTRODUCCIÓN}

El etanol se produce por la fermentación de los azúcares contenidos en la materia orgánica de las plantas, fundamentalmente la caña de azúcar y el maíz, con enzimas o levaduras y, debido a esta fuente de obtención, en numerosos ámbitos se denomina bioetanol. En este proceso se obtiene el alcohol hidratado, con un contenido aproximado de $5 \%$ de agua que, tras ser deshidratado, se puede utilizar como combustible. De este etanol mezclado con la gasolina, se obtiene un biocombustible de alto poder energético y con características muy similares a la gasolina pero con una importante reducción de las emisiones contaminantes en los motores tradicionales de combustión. Las legislaciones de la mayoría de los países,

\footnotetext{
(•) Trabajo recibido el día 15 de marzo de 2010 y aceptado en su forma final el día 25 de junio de 2010 .

* Departamento de Ingeniería Mecánica, Energética y de los Materiales, Escuela Técnica Superior de Ingenieros Industriales y Telecomunicaciones, Universidad Pública de Navarra, Calle Arrosadía, s/n, 31006 Pamplona, e-mail: carlos.berlanga@unavarra.es. ** Departamento de Ciencia e Ingeniería del Terreno y de los Materiales, Escuela Técnica Superior de Náutica, Universidad de Cantabria, Germán Gamazo, 1, 39004 Santander.
} 
permiten utilizar etanol mezclado con gasolina en concentraciones del 5 y $10 \%$, E5 y E10 respectivamente, que no requieren modificaciones en los motores actuales. Concentraciones más elevadas, implica que se debe disponer de un vehículo flexible, con un depósito, motor y sistema de combustible único y capaz de funcionar con gasolina y etanol, solos o mezclados en cualquier proporción. Por otra parte, el etanol también se utiliza en forma de aditivo de la gasolina como etil-tercbutil éter (ETBE).

El conocimiento de las reacciones químicas que pueden producirse entre el etanol y los materiales que forman parte de los motores de combustión, no es reciente, ya que a finales del siglo XIX, se propuso el etanol para su uso como combustible en los primeros motores de explosión de ciclo Otto ${ }^{[1]}$, pero no fue hasta la década de los 70 del siglo pasado, cuando Brasil empezó a utilizarlo, aprovechando que se obtenía como subproducto de la caña de azúcar.

Hasta hace pocos años no se había planteado seriamente su utilización de forma significativa a nivel mundial; el principal motivo del renovado interés por el etanol ha sido su consideración como combustible neutro, a efecto de emisiones de dióxido de carbono, monóxido de carbono y gases sulfurados, entre otros ${ }^{[2]}$. Un estudio reciente, llevado a cabo para estudiar el grado de contaminación que produce el empleo de combustibles mezclas de gasolina con distintos combustibles de origen biológico, y en distintas proporciones, ha demostrado que los niveles de $\mathrm{NO}_{\mathrm{x}}(\mathrm{g})$ y $\mathrm{CO}(\mathrm{g})$ se reducen notablemente al aumentar la proporción de combustible no fósil en la mezcla ${ }^{[3]}$. Por otra parte, los países europeos y Estados Unidos, pretenden disminuir su dependencia de los combustibles fósiles ${ }^{[4]}$. En relación con este objetivo, el Parlamento Europeo ha propuesto incrementar el porcentaje de consumo total de biocumbustibles hasta un 5,75 \% para el año $2010^{[5]}$. Finalmente, hay que destacar que su uso como aditivo en gasolina, incrementa el octanaje de ésta ${ }^{[6}$ y 7$]$, por tanto, el rendimiento de los motores.

Las propiedades físico químicas del etanol y de la gasolina son muy distintas, y su corrosividad potencial apenas se ha estudiado de forma comparativa. Los biocarburantes en base alcohol, tienen una elevada miscibilidad con el agua y además, se puede producir la oxidación parcial de sus componentes por su exposición a la atmósfera, aumentando su acidez siendo, por tanto, potencialmente corrosivos. Por otra parte, el etanol deshidratado también puede ocasionar problemas de corrosión, debido principalmente a su naturaleza higroscópica. Además, durante su almacenaje, el etanol se puede oxidar a ácido acético y aumentar su acidez, con el consiguiente riesgo corrosivo.

El origen de la presencia de agua en el etanol es diverso; generalmente proviene de su obtención, al contener $5 \%$ de agua azeotrópica que no se elimina en los procesos normales de destilación. Para evitarlo se emplean distintas técnicas, entre las que destacan la utilización reciente de microorganismos ${ }^{[8]}$. Este etanol hidratado ha sido muy utilizado en Brasil mezclado con gasolina, comprobándose que, en ausencia de inhibidores adecuados, las piezas de los vehículos estaban sometidas a problemas importantes de corrosión $^{[9]}$. Por otro lado, hay que destacar la aparición de fallos recientes en componentes metálicos de fábricas de etanol de Estados Unidos, que se han asociado al fenómeno de corrosión bajo tensión. Éste ha sido un hecho sorprendente, ya que en Brasil no había existido este problema, de lo que puede deducirse que la causa está relacionada con el diferente modo de obtención del etanol.

De todo esto, se puede concluir, que todos los materiales metálicos que están en contacto con el etanol, desde su obtención, hasta su utilización en los vehículos, son susceptibles de sufrir corrosión. Además, la sustitución de un combustible tan extendido, como es la gasolina, conlleva un proceso largo y complejo, ya que involucra a muchas industrias y normativas. El objetivo de este trabajo, ha sido realizar una revisión sobre los problemas existentes y soluciones planteadas en relación con la corrosión que experimentan distintos sistemas metálicos, producida por el etanol y sus mezclas con gasolina.

\subsection{Normativa que regula la composición del etanol como combustible}

Debido a su influencia en la corrosividad del etanol, es importante conocer los límites impuestos en las normas para el contenido en agua, $\mathrm{pH}-\mathrm{pHe}$, ácido acético y cloruros en el etanol. Para ello, se ha analizado la información que proporcionan las normas existentes para el etanol deshidratado en Estados Unidos ${ }^{[10]}$, Brasil $^{[11]}$ y Europa ${ }^{[12]}$. Las especificaciones para la utilización de etanol se compendian en la norma Europea de Gasolinas EN 228. En España se encuentra recogida en la Directiva 2003/17/CE relativa a la calidad de las gasolinas y gasóleo y en el Real Decreto R.D. 61/2006 de las especificaciones y uso de biocarburantes.

Al ser Brasil el país que más emplea etanol como combustible, incluso de forma hidratada en la proporción 95,6 \% etanol con 4,4 \% de agua, establece una norma específica para el alcohol hidratado. En la tabla I se presenta la composición química y propiedades físico-químicas del etanol, atendiendo a distintas normativas vigentes. En Brasil, se ha establecido un contenido mínimo muy alto de etanol, 99,3\%, considerándose innecesario establecer límites para otros compuestos, como el agua y el ácido acético. El máximo contenido en agua del bioetanol en Europa es de un 
REVISIÓN Y ESTADO DEL ARTE DE LA CORROSIVIDAD DEL ETANOL Y SUS MEZCLAS CON GASOLINA REVIEW AND STATE-OF-THE-ART OF THE CORROSIVITY PRODUCED BY ETHANOL AND THEIR MIXTURES WITH GASOLINE

Tabla I. Composición química y propiedades físicas del etanol según la normativa vigente

Table I. Chemical composition and physical properties of ethanol according to the actual standards

\begin{tabular}{|c|c|c|c|c|}
\hline & $\begin{array}{c}\text { ASTM } \\
\text { D4806 } \\
\text { (EE.UU.) }\end{array}$ & $\begin{array}{c}\text { Brasil } \\
\text { Etanol } \\
\text { hidratado }\end{array}$ & $\begin{array}{c}\text { Brasil } \\
\text { Etanol } \\
\text { deshidratado }\end{array}$ & $\begin{array}{c}\text { PrEn } \\
15376 \\
\text { (Europa) }\end{array}$ \\
\hline Etanol (\% v/V) Min & 92,1 & 99,3 & 92,6 & 98,7 \\
\hline Agua $(\% \mathrm{v} / \mathrm{V})$ & 1 & - & - & 0,3 \\
\hline Cloruros (mg/l) & 40 & - & 1 & 40 \\
\hline $\mathrm{pHe}$ & $6,5-9$ & - & $6-8$ & - \\
\hline $\begin{array}{l}\text { Acidez (como ácido } \\
\text { acético) }(\% \mathrm{~m} / \mathrm{V})\end{array}$ & 0,007 & 0,003 & 0,003 & 0,007 \\
\hline Conductividad $(\mu \mathrm{S} / \mathrm{m})$ & - & 500 & 500 & - \\
\hline
\end{tabular}

0,3\%, mientras que en Estados Unidos, según la norma ASTM D 4806, es de un 1,0\% [10]

El nivel de acidez se cuantifica de dos formas: $\mathrm{pHe}$ y $\mathrm{pH}$. El pHe indica el contenido en ácidos fuertes, y se aplica cuando el contenido en etanol, es como mínimo el 70 \% del volumen total de la disolución. El pHe permitido en Estados Unidos y Europa es similar, y varía entre los valores de 6,5 y 9. En Brasil se emplea el $\mathrm{pH}$, oscilando sus valores entre 6 y 8 . La acidez, medida en función de la cantidad de ácido acético, varía de 70 ppm en Europa y Estados Unidos a 30 ppm en Brasil; el nivel de cloruros oscila entre los valores de $1 \mathrm{ppm}$ en Brasil, hasta 40 ppm de Estados Unidos y Europa.

Por otra parte, la legislación exige desnaturalizar el etanol para evitar su posible uso como bebida alcohólica, y para ello se emplea la propia gasolina que se usa en las mezclas, aunque también se pueden utilizar diversos productos químicos.

Finalmente, la Agencia Federal de Energías Renovables de Estados Unidos, Renewable Fuels
Association ${ }^{[13]}$, recomienda que se añadan inhibidores de corrosión al etanol utilizado como combustible, en una concentración suficiente para evitar la corrosión. Estos inhibidores ajustan el pHe y, por tanto, controlan la actividad de los ácidos fuertes.

\section{REVISIÓN DE LOS ESTUDIOS DE LA CORROSIÓN EN ALEACIONES PRODUCIDA POR LAS MEZCLAS ETANOL-GASOLINA}

La corrosión producida por disolventes orgánicos ha sido extensamente estudiada, debido a su importancia técnica e industrial ${ }^{[14-21]}$. Los mecanismos de corrosión asociados a líquidos orgánicos son más complejos y menos conocidos que los que se producen en disoluciones acuosas. Los alcoholes, aunque son disolventes próticos como el agua, presentan diferentes propiedades físico-químicas ${ }^{[22]}$. En la tabla II

Tabla II. Propiedades físico-químicas del agua y de diferentes líquidos orgánicos

Table II. Physical-chemical properties of water and different organics liquids

\begin{tabular}{|c|c|c|c|c|}
\hline Medio & $\begin{array}{c}\text { Constante } \\
\text { Dieléctrica a } 25^{\circ}\end{array}$ & $\begin{array}{c}\text { pH (Valor de } \\
\text { C neutralización) }\end{array}$ & $\begin{array}{c}\text { Solubilidad del } \\
\text { Oxígeno }\left(\mathrm{cm}^{3} \text { a }\right. \\
\left.1 \text { atm } / 25^{\circ} \mathrm{C}\right)\end{array}$ & $\begin{array}{c}\text { Conductividad } \\
\text { específica } \\
\left(\mathrm{Ohm}^{-1} \mathrm{~cm}^{-1}\right)\end{array}$ \\
\hline Agua & 78,5 & 7,00 & 0,022 & $5,6 \times 10^{-1}$ \\
\hline Metanol & 32,6 & 8,35 & 0,227 & $1,6 \times 10^{-1}$ \\
\hline Etanol & 24,3 & 9,55 & 0,221 & $4,3 \times 10^{-2}$ \\
\hline Ácido Fórmico & 48,0 & 3,10 & 0,047 & $2,8 \times 10^{-2}$ \\
\hline Ácido Acético & 6,4 & 7,70 & 0,180 & $2,8 \times 10^{-3}$ \\
\hline
\end{tabular}


se presentan distintas propiedades físico-químicas del agua y diferentes líquidos orgánicos: metanol, etanol, ácido fórmico y ácido acético. En dicha tabla se observa que, para alcanzar la neutralidad en el etanol, es necesario un valor de $\mathrm{pH}$ de 9,5. Los valores de solubilidad del oxígeno en etanol y metanol son similares, y de un orden de magnitud mayor que el del agua. Por tanto, la disponibilidad del oxígeno, para reacciones de corrosión, es mayor en el caso del etanol y sus soluciones respecto al agua. Por otra parte, la conductividad del etanol, es de un orden de magnitud menor que la del agua, lo que favorece la disminución de la velocidad de las reacciones de corrosión.

La alta resistividad del etanol, dificulta el empleo de ensayos electroquímicos que ofrezcan cinéticas de corrosión directas, debido a la fuerte caída óhmica que provoca.Tradicionalmente, esta dificultad experimental se superaba añadiendo sales, como por ejemplo $\mathrm{LiClO}_{4}$ a las soluciones etanólicas, para aumentar su conductividad. Recientemente, Gui y Sridhar ${ }^{[23]}$ han demostrado que es posible utilizar microelectrodos para resolver este problema.

Otro aspecto importante a considerar, es la naturaleza higroscópica del etanol en comparación con otros combustibles. En la tabla III se presentan los resultados obtenidos por Nakajima et al.[24]. Resulta llamativo que, tras 30 días de exposición en una atmósfera con una humedad relativa del $100 \%$, la absorción de agua por el etanol es significativamente mayor respecto a la gasolina y la mezcla E10, siendo ésta una mezcla de $10 \%$ de etanol anhidro y $90 \%$ de gasolina.

La investigación sobre la corrosión que produce el etanol no es reciente, ya que antes de su desarrollo como combustible, se utilizaba en la industria como refrigerante. En estas aplicaciones, ya se observó el potencial corrosivo del etanol comprobándose que, los metales pierden su capacidad de pasivarse, durante los ensayos de polarización anódica ${ }^{[25}$ y 26]. En los procesos de producción, utilización y distribución del etanol se emplean distintos sistemas metálicos, y se puede producir una casuística muy elevada de tipos de corrosión. Ahora bien, apenas existen estudios metodológicos que consideren las numerosas variables involucradas por lo que, a continuación, se presentan las más determinantes que influyen en la corrosividad de las mezclas etanol-gasolina.

\subsection{Efecto del contenido de etanol}

Una de las variables principales que se tienen en cuenta en la investigación experimental, es el contenido de etanol en sus mezclas con gasolina, y su efecto corrosivo sobre los materiales metálicos con los que entra en contacto. En principio, es esperable que, un incremento del porcentaje de etanol, provoque un incremento de impurezas, agua, cloruros, etc., así como de conductividad eléctrica de la mezcla final del combustible y, por tanto, que se favorezca el mecanismo de corrosión electroquímica. Desafortunadamente, no existen estudios sistemáticos que hayan comprobado la relación entre el incremento del contenido de etanol y el aumento de corrosividad del combustible final.

Nie et al. ${ }^{[27]}$, determinaron la resistencia a la corrosión de varias aleaciones metálicas, aleaciones de aluminio $\mathrm{Al} 6061$ y Al 319, acero inoxidable AISI 304 y fundición gris 2500, en los combustibles E30, E85 y E100 en etanol hidratado $\left(5 \% \mathrm{H}_{2} \mathrm{O}\right)$. El contenido en etanol apenas influye en el potencial de corrosión y en la resistencia a la polarización del acero inoxidable austenítico AISI 304, tal y cómo se observa en la figura 1. La resistencia a la polarización de la fundición gris es, en presencia de todos los combustibles estudiados, más baja que la observada para las aleaciones de aluminio, aunque aumenta de $10,7 \times 10^{3} \mathrm{ohm} / \mathrm{cm}^{2}$ en E30 a $56,9 \times 10^{3} \mathrm{ohm} / \mathrm{cm}^{2}$ en la mezcla E100. Finalmente, estos investigadores, destacaron que la aleación de aluminio Al 1319 experimentaba velocidades de corrosión mayores que la aleación Al 6061; este comportamiento, lo asociaron a la presencia de fases ricas en silicio en la aleación 1319, que actuaban como zonas catódicas, favoreciendo la formación de pilas de corrosión.

Tabla III. Higroscopicidad de la gasolina, etanol y E10 [24]

Table III. Hygroscopicity of gasoline, ethanol and E10 [24]

\section{Combustible Contenido en agua antes Contenido en agua (Vol. \%) después de 30 de la exposición (Vol. \%) 30 días a $20{ }^{\circ} \mathrm{C}$ y $100 \%$ de humedad relativa}

\begin{tabular}{crr}
\hline Gasolina & 0,02 & 0,10 \\
E 10 & 0,03 & 0,72 \\
Etanol & 0,21 & 51,75 \\
\hline
\end{tabular}




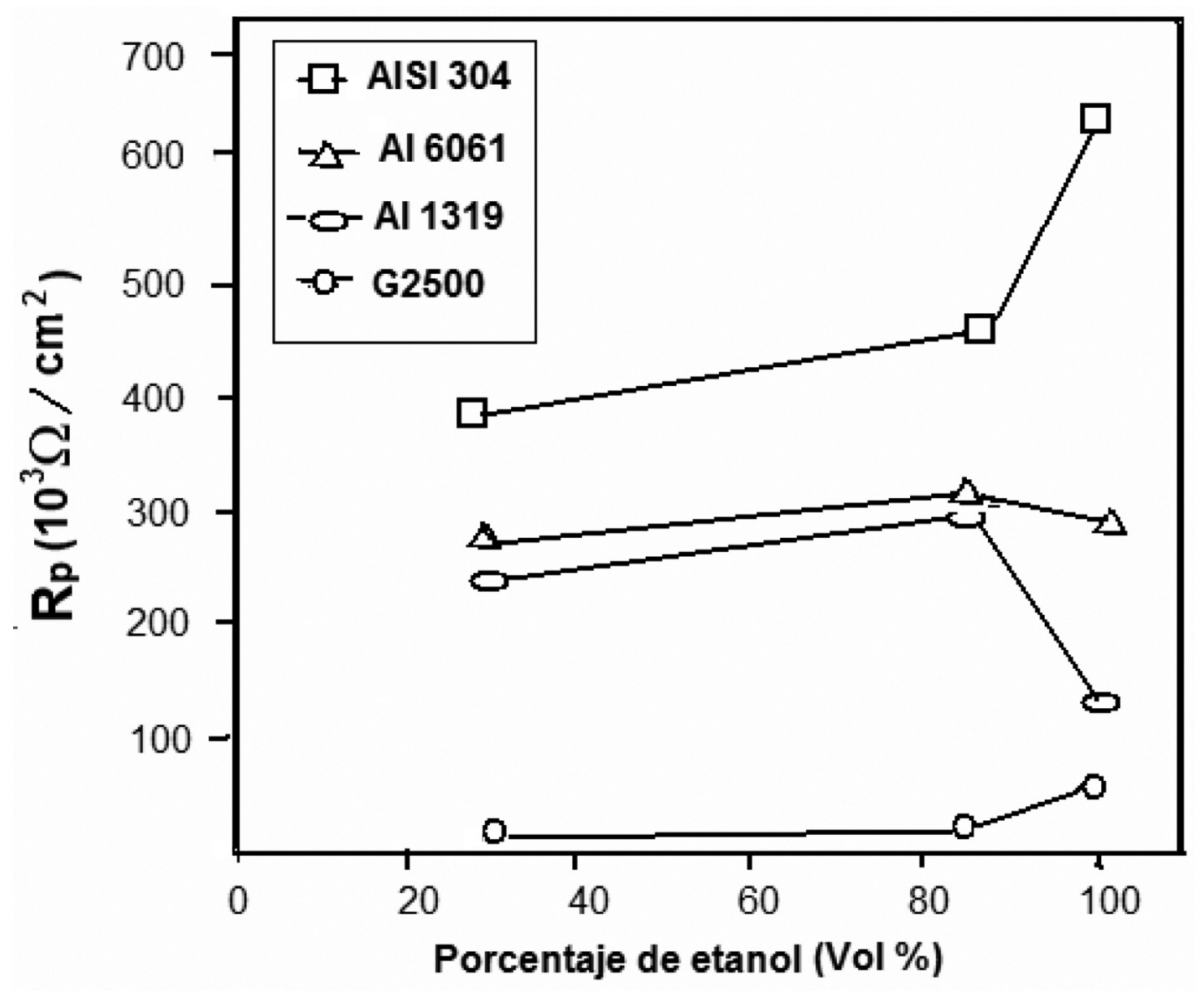

Figura 1. Relación entre la resistencia a la polarización de cuatro aleaciones (aleaciones de aluminio Al 6061 y Al 1319, acero inoxidable AISI 304 y fundición gris G2500) y el contenido en etanol. Tomada de Nie et al. ${ }^{[27]}$.

Figure 1. Relationship between polarization resistance of four alloys tested (AI 6061, Al 1319, AISI 304 and grey cast iron G2500) and ethanol content. Taken from Nie et al. ${ }^{[27]}$.

\subsection{Efecto del contenido en agua}

El papel del agua es determinante en el estudio de la corrosividad de las mezclas etanol-gasolina y, de hecho, es una de las variables que marca la calidad del etanol como biocombustible. Esto se debe tanto a su papel como disolvente de impurezas inorgánicas como a su protagonismo en los mecanismos de corrosión. El etanol y el agua son solubles en todas las proporciones, mientras que el contenido máximo de agua que puede contener la gasolina es de sólo 0,19 \%. Se ha calculado que el combustible E90, $90 \%$ etanol, puede contener hasta un máximo de $0,5 \% \mathrm{H}_{2} \mathrm{O}$ antes que sus fases se separen, lo cual puede favorecer el aumento de la velocidad de corrosión ${ }^{[28]}$.

Los contenidos elevados de agua, en las mezclas etanol-gasolina, promueven la corrosión de los distintos sistemas metálicos empleados en los sistemas de combustión de vehículos, observándose una fuerte dependencia con la temperatura de servicio, en particular en aleaciones de aluminio, así como en el comportamiento de materiales poliméricos ${ }^{[29]}$.
V.P. Persiantseva et al. ${ }^{[25]}$, realizaron ensayos de inmersión de un acero al carbono, una aleación de aluminio y cobre de alta pureza, durante 15 días en soluciones etanol/agua, con distintas concentraciones de etanol. Observaron que las velocidades de corrosión en etanol puro y agua son similares, alcanzándose las cinéticas de corrosión más elevadas para la mezcla formada por porcentajes volumétricos idénticos de etanol y agua. Las curvas de polarización electroquímica de la aleación de aluminio D-16, (Fig. 2) en soluciones etanol/agua, muestran zonas de pasividad amplias, especialmente para las soluciones con contenido de etanol que varía entre el 0 y el $75 \%$. Las densidades de corriente de pasivación oscilan entre márgenes muy grandes, alcanzando el valor de $66 \mathrm{~mA} / \mathrm{cm}^{2}$ para la mezcla $75 \%$ etanol - $25 \%$ agua. Al mismo tiempo, con la mezcla formada por un $50 \%$ de agua en etanol, el potencial de corrosión de la aleación aumenta, mientras que el cobre y especialmente los aceros al carbono, desplazan su potencial de corrosión hacia valores más activos. 


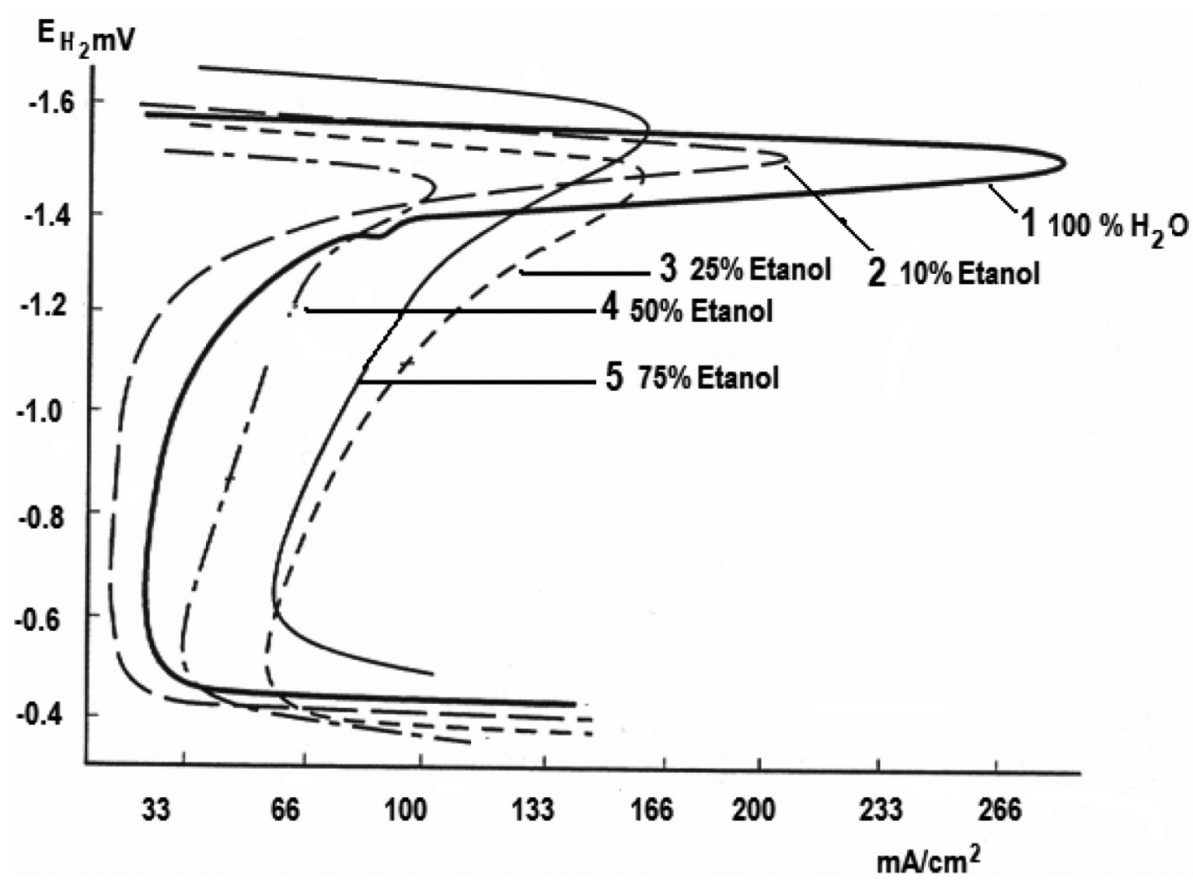

Figura 2. Curva de polarización de la aleación de aluminio D16 en soluciones etanol/agua (1: $100 \% \mathrm{H}_{2} \mathrm{O} ; 2$ : $10 \%$ etanol; 3: $25 \%$ etanol; 4: $50 \%$ etanol; $5: 75 \%$ etanol). Tomada de Persiantseva et al[25].

Figure 2. The polarization curve of $D-16$ aluminum alloy in water/etanol solutions (1: $100 \% \mathrm{H}_{2} \mathrm{O}$; 2: $10 \%$ ethanol; 3: $25 \%$ ethanol; $4: 50 \%$ ethanol; 5: $75 \%$ ethanol). Taken from Persiantseva et al[25].

Kabasakaloglu et al. ${ }^{[30]}$, obtuvieron curvas de polarización anódica con mezclas etanol-agua en un medio al que se añadió $\mathrm{H}_{2} \mathrm{SO}_{4} 0,1 \mathrm{M}$ observando que, cuando el contenido de agua aumentaba, los valores de las densidades de corrientes anódicas eran más elevadas. También comprobaron que en una solución de etanol puro, la curva no tiene picos de pasivación. Sridhar et al. ${ }^{[31]}$, realizaron curvas de polarización cíclicas con un acero al carbono en mezclas etanol/agua, y con diferentes contenidos en agua $\left(0-5 \% \mathrm{H}_{2} \mathrm{O}\right)$, recogiendo sus resultados con y sin corrección de la caída óhmica. Observaron que, en las curvas con corrección óhmica, la densidad de corriente anódica máxima aumentaba con el contenido de agua de la mezcla.

Traldi et al. ${ }^{[32]}$, estudiaron el comportamiento frente a la corrosión de dos aleaciones $\mathrm{Si}-\mathrm{Al}$-Cu hipereuctécticas, obtenidas mediante proyección térmica, y una fundición gris, en etanol hidratado con un $1,5 \% \mathrm{H}_{2} \mathrm{O}$, mediante ensayos de polarización anódica. Concluyeron que las dos aleaciones de aluminio presentaban mejor comportamiento frente a la corrosión respecto a la fundición gris. Gui y Sridhar ${ }^{[23]}$, estudiaron la resistencia a la corrosión de un acero al carbono CS 1010 en dos mezclas de etanol-agua en proporciones de $1 \%$ y $8 \%$ de agua. Comprobaron que el potencial de circuito abierto del acero en el etanol que contenía un $8 \%$ en agua, disminuía aproximadamente $150 \mathrm{mV}$ en relación al potencial que presentaba el acero en el electrolito que contenía $1 \%$ de agua. Este hecho, lo asociaron tanto a un incremento de la disolución anódica del hierro, como a un incremento de la corriente de oxidación del agua. Además afirmaron que la corrosión localizada en el etanol con $8 \%$ de agua, era mucho más severa que con $1 \%$ de agua.

\subsection{Efecto de la variación del contenido en ácidos}

De Anna ${ }^{[19]}$, evaluó el comportamiento frente a la corrosión de una fundición y un acero inoxidable austenítico AISI 304L en soluciones alcohólicas con pequeñas adiciones de cloruros, $1,10^{-1}$ a $1,10^{-5} \mathrm{M}$, en forma de $\mathrm{HCl}$. Mediante la obtención de curvas de polarización potenciodinámicas, concluyeron que los cloruros aumentaban la velocidad de corrosión en ambos materiales, especialmente para concentraciones comprendidas entre $1,10^{-2} \mathrm{M}$ y $1,10^{-3} \mathrm{M}$.

Kabasakaloglu et al. ${ }^{[30]}$, estudiaron el comportamiento de un acero al carbono, variando la 
concentración de $\mathrm{H}_{2} \mathrm{SO}_{4}$ en etanol hidratado (4 \%). El potencial y la densidad de corriente crítica de pasivación disminuían, con el aumento de concentración del ácido mientras que, para concentraciones bajas $\left(0,001 \mathrm{M} \mathrm{H}_{2} \mathrm{SO}_{4}\right)$, el metal se pasivaba. Concluyeron que la velocidad de corrosión depende notablemente de la conductividad de la solución, directamente proporcional a la concentración de ácido. Traldi et al. ${ }^{[32]}$, ensayaron una aleación hipereutéctica de Al-Si y una fundición gris en etanol, con una concentración $1 \mathrm{mM}$ de $\mathrm{H}_{2} \mathrm{SO}_{4}$ mediante espectroscopia de impedancia electroquímica. Resaltaron que la adición del ácido causaba la disolución inicial del óxido presente en la superficie, impidiendo su posterior formación. Además, la aleación de aluminio presentaba mejor resistencia a la corrosión que la fundición gris.

\subsection{Efecto combinado de varios factores}

Jahnke y Schoenborn ${ }^{[33]}$, ensayaron el efecto de la mezcla etanol-agua-ácido fórmico, en dos aceros al carbono y dos aceros aleados con cromo y molibdeno, observando que la velocidad de corrosión aumentaba considerablemente con el contenido de ácido fórmico. Goncalves et al. ${ }^{[34]}$, analizaron el comportamiento de un acero al carbono en soluciones de etanol, y con diferentes concentraciones de agua y ácido acético simultáneamente, mediante ensayos de inmersión durante 30 días a $25^{\circ} \mathrm{C}$ y ensayos electroquímicos. Observaron que velocidad de pérdida de peso, disminuía proporcionalmente con el aumento de concentración de ácido acético en una solución de etanol 0,1M, concluyendo que el ácido acético inhibía el proceso de corrosión del acero al carbono en presencia de esta mezcla.

De Anna ${ }^{[19]}$, observó que adiciones crecientes de agua en soluciones alcohólicas que contenían $\mathrm{HCl}$ tienen un efecto pasivante sobre el acero inoxidable austenítico AISI 304.

Tanaka et al. ${ }^{[35]}$, estudiaron el efecto de la variación del contenido de agua, ( $1-80 \%)$, en etanol con un $20 \%$ de $\mathrm{HCl}$. Los resultados indicaron que, para concentraciones de entre 4 y $6 \%$ de agua en etanol, la velocidad de corrosión disminuía de forma considerable; para concentraciones entre 6 y $32 \%$ de agua, la velocidad de corrosión se mantiene aproximadamente constante, y a partir de un $32 \%$ vuelve a aumentar. La morfología de corrosión predominante es de tipo uniforme, para todos los porcentajes de agua en etanol, excepto para las concentraciones que oscilan entre un 4 y $6 \%$, y en donde predomina la corrosión por picaduras.

E. Cavalcanti et al. ${ }^{[36]}$, estudiaron el efecto del contenido de agua, sulfatos y $\mathrm{pH}$ en el comportamiento frente a la corrosión de un acero al carbono AISI 1010 en disoluciones etanólicas. Realizaron ensayos de inmersión durante 78 días a $25^{\circ} \mathrm{C}$ y observaron que la morfología de corrosión predominante era por picaduras, para una concentración de sulfatos que oscilaba entre 0,5 y $2 \mathrm{mg} / \mathrm{l}$, un rango de $\mathrm{pH}$ de 5,6 - 7,9 y un contenido en $\mathrm{H}_{2} \mathrm{SO}_{4}$ variable entre $6,1 \times 10^{-3} \mathrm{mg}$ y $1,10 \times 10^{-2} \mathrm{mg} / \mathrm{ml}$. Finalmente, destacaron la necesidad de mantener el pH entre 8 - 9, con objeto de minimizar la velocidad de corrosión. Los productos de corrosión que se formaron también dependían del contenido de agua. En etanol anhidro, observaron principalmente la aparición de $\mathrm{FeO}(\mathrm{OH})$ y también $\mathrm{Fe}_{2} \mathrm{O}_{3}$. Para una concentración de agua de un $20 \%$, los productos hallados fueron principalmente $\mathrm{Fe}_{2} \mathrm{O}_{3}$ y $\mathrm{Fe}_{3} \mathrm{O}_{4}$.

Singh y Singh ${ }^{[37]}$, estudiaron el comportamiento de un acero inoxidable austenítico AISI 304 en diferentes alcoholes anhidros, etanol, isopropanol, t-butanol y con diferentes concentraciones de $\mathrm{H}_{2} \mathrm{SO}_{4}$ $(0,001-1 \mathrm{M})$. La velocidad de corrosión era mayor en el etanol respecto a los otros dos alcoholes, Además, el acero presentaba corrosión por picaduras para altas concentraciones de ácido sulfúrico en etanol.

Sekine et al. ${ }^{[38]}$, estudiaron el comportamiento frente a la corrosión de un acero inoxidable ferrítico AISI 430 para diferentes concentraciones en etanol de agua (0-100 \%) y ácido sulfúrico 0,1M. La velocidad de corrosión observada aumentaba cuando disminuía la concentración de etanol, debido a la mayor conductividad de la mezcla, y a la transferencia de carga de las disoluciones acuosas.

\subsection{Efecto de los inhibidores}

Se han estudiado varios tipos de inhibidores para hacer disminuir la velocidad de corrosión en etanol. Codling ${ }^{[9]}$, comprobó que el aditivo "Promaz 8027", compuesto por una mezcla de glicoles y aminas, en una concentración de 0,02 \%, inhibía notablemente la corrosividad del etanol hidratado.

Wiggle et al. ${ }^{[39]}$, estudiaron un acero al carbono y una aleación Zamak mediante ensayos electroquímicos en etanol, que contenía $10 \%$ de agua, 10 ppm de $\mathrm{NaCl}$ y 100 ppm de ácido acético y en presencia de 12 inhibidores diferentes, cuyas concentraciones oscilaban entre 0,1 y $0,5 \%$. Concluyeron que ninguno de ellos proporcionaba una adecuada protección para exposiciones largas, y que había que mejorar su formulación para aumentar su poder inhibidor.

Janke y Schoenborn ${ }^{[33]}$, utilizaron morfolina, piperazina y hexametilendiamina, en una concentración de 200 ppm, y observaron cómo la adición de inhibidores en base amina al metanol o etanol, provocaba una disminución significativa de la velocidad 
de corrosión. Además, el aditivo morfolina, también disminuía el efecto corrosivo del etanol contaminado con 100 ppm de cloruros.

Gui y Sridhar ${ }^{[23]}$, estudiaron el efecto inhibidor del $\mathrm{NH}_{4} \mathrm{OH}$ para evitar la Corrosión Bajo Tensión de un acero al carbono en etanol, observando que la adición de 37,5 ppm de $\mathrm{NH}_{4} \mathrm{OH}$ generaba corrientes anódicas pequeñas, desplazándose hacia valores más nobles el potencial de corrosión.

\section{COMPATIBILIDAD DE MATERIALES METÁLICOS UTILIZADOS EN COMPONENTES PARA AUTOMÓVILES CON MEZCLAS ETANOL - GASOLINA}

Para comprobar la compatibilidad de los materiales metálicos, utilizados en componentes de vehículos en mezclas etanol-gasolina, se han realizado ensayos de inmersión, ensayos en autoclave y ensayos de campo utilizando vehículos. Los ensayos de inmersión son muy útiles ya que, aunque no simulan bien las condiciones de servicio de los materiales, se pueden usar como paso previo a la selección de materiales, sin que supongan un coste elevado. Los ensayos en autoclave, simulan las condiciones en servicio de los motores y son regulados por normas provenientes del sector de la automoción. Los ensayos de campo, al emplear los propios vehículos, ofrecen los resultados más válidos, pero presentan la desventaja ya que se ajustan a las condiciones de servicio pero la desventaja de su elevado coste. Los pioneros en realizar investigaciones con este tipo de ensayos fueron tanto universidades como empresas de automoción brasileñas, durante la década de los años $80{ }^{[40-46]}$. Wolynec y Tanaka ${ }^{[40]}$, realizaron ensayos mediante la utilización de vehículos alimentados con etanol hidratado durante $30.000 \mathrm{~km}$. Extrajeron todas las piezas que estaban en contacto con el combustible, evaluando el daño que habían sufrido. Destacaron la corrosión severa por picaduras detectada en el recubrimiento de zinc del depósito de combustible, así como corrosión de los carburadores, fabricados con la aleación Al-Zn, Zamak. Para solucionar estos problemas, se evaluaron 18 recubrimientos distintos y aplicados sobre un acero al carbono AISI $1020^{[41]}$. Los recubrimientos de óxido de aluminio, dacromet, níquel y estaño ofrecieron una buena protección al metal y, en particular, los de níquel y estaño aumentaban mucho su capacidad protectora utilizándolos con una capa intermedia de cobre. Por otro lado, los recubrimientos de aluminio, plomo, fosfatos, zinc y zinc/aluminio ofrecían poca resistencia a la corrosión en etanol.

El gobierno de Australia ha promovido la investigación para estudiar el efecto del etanol, tanto en motores de automoción como en otro tipo de motores ${ }^{[47-50]}$. Comprobaron que, aunque para la mezcla E10 no existían problemas de compatibilidad, si los había para la mezcla E20, debido al desprendimiento de los óxidos superficiales de las piezas metálicas, quedando aquéllos entre las superficies móviles de los componentes. Esta situación podría producir su desgaste acelerado y, por lo tanto, un pésimo funcionamiento del motor. En un reciente trabajo, B. Jones et al. ${ }^{[51]}$, ensayaron quince materiales, aleaciones base hierro, cobre y aluminio, además de diferentes recubrimientos. Los combustibles que utilizaron fueron fuel C, fuel C con E10, Fuel C con E20 y gasolina. Los ensayos se realizaron por inmersión, para estudiar el efecto corrosivo de estos combustibles en estado líquido y gaseoso, a la temperatura de $45^{\circ} \mathrm{C}$ durante 2.016 h. La conclusión principal fue que, únicamente la aleación Zamak 5, presentaba unas velocidades de corrosión inaceptables, tras su exposición a las mezclas E10 y E20, debido a la elevada presencia de corrosión por picaduras y posterior pérdida de productos de corrosión. Las catorce aleaciones restantes estudiadas se consideraron compatibles con los combustibles utilizados.

\section{CORROSIÓN BAJO TENSIÓN DE MATERIALES METÁLICOS EN MEZCLAS DE ETANOL - GASOLINA}

La existencia del fenómeno de corrosión bajo tensión, CBT, de aleaciones metálicas en medios orgánicos no es nueva; por ejemplo, se ha observó la existencia de CBT en aceros al carbono en ambientes ricos en amoníaco inherentes a las industrias de fer-

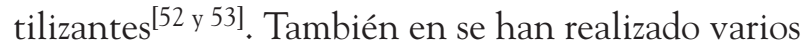
trabajos con aleaciones no férreas, en base titanio y zirconio en contacto con soluciones alcohólicas, empleándose en la mayoría de los ensayos metanol como reactivo de ataque ${ }^{[54-59]}$.

El interés por investigar la corrosión bajo tensión en soluciones etanólicas es más reciente, debido al empleo potencial de estas mezclas como combustible. La celebración de un Forum en Richmond, Estados Unidos, en el año 2003, organizado por empresas relacionadas con el etanol, se puede considerar el punto de inicio del reconocimiento de la existencia de este problema, ya que los productores y distribuidores de etanol describieron sus experiencias en planta, relacionadas con los fallos de componentes causados por la corrosión bajo tensión, CBT. El Instituto Americano del Petróleo, American Petroleum Institute, API ${ }^{[60]}$, recogió todas las experiencias en un primer informe, que fue reeditado posteriormente ${ }^{[61]}$. En estos informes se resaltó la impor- 
tancia industrial del problema debido al fallo de componentes metálicos por CBT en menos de un año de vida operativa, estimándose pérdidas económicas de 1 millón de dólares hasta ese momento. Las conclusiones más importantes de estos informes fueron las siguientes:

- La existencia de fallos ocurría principalmente en el almacenamiento y distribución del etanol antes de su mezcla con la gasolina.

- La identificación de los fallos en zonas de elevadas tensiones residuales, por ejemplo, zonas de soldaduras sin tratamiento térmico posterior.

- La necesidad de relacionar de una forma clara las propiedades físico químicas del etanol, $\mathrm{pH}$, oxígeno disuelto, cloruros, ácidos etc., con el efecto que produce en la susceptibilidad a la corrosión de distintos sistemas metálicos.

Los trabajos posteriores a este informe han tenido como objetivo principal identificar las condiciones que producen CBT, así como describir el mecanismo de fractura.

Shridar et al. ${ }^{[31]}$, realizaron un extenso programa de ensayos, para detectar los factores más importantes que influyen en el fenómeno de CBT de un acero al carbono en presencia de etanol. Observaron que la susceptiblidad a la CBT se incrementa con la cantidad de oxígeno disuelto, variable más influyente ya que, en los ensayos sin aireación, no se observó corrosión bajo tensión. Asimismo, influía negativamente el contenido de cloruros y ácido acético.

Recientemente Lou et al. ${ }^{[62]}$, investigaron la susceptibilidad a la corrosión bajo tensión de un acero al carbono API 5LX-65, mediante ensayos de deformación a baja velocidad, Strain Slow Rate Testing, SSRT, tomando como variables el contenido en agua, presencia de cloruros, e inhibidores de la corrosión, en particular hidracina, $\mathrm{pHe}$, oxígeno disuelto, existencia de pares galvánicos, así como la velocidad de deformación. Todos esto factores influían en la iniciación y crecimientos de las grietas siendo el modo de fractura transgranular idéntico al que observaron Shirdar et al. ${ }^{[31]}$. También destacaron que, un aumento del contenido en agua, provocaba un cambio en el tipo de corrosión, pasando de corrosión bajo tensión a corrosión por picaduras. Concluyeron que la probabilidad de aparición del fenómeno de la corrosión bajo tensión en los materiales ensayados, depende íntimamente del contenido en cloruros presentes en el etanol comercial. Lou et al. ${ }^{[63]}$, estudiaron con detalle el mecanimo de corrosión de un acero al carbono en etanol. Observaron que, una vez iniciada la grieta, existe una competición entre la disolución anódica y la repasivación del sistema metálico, hecho que determina la velocidad de crecimiento de la grieta. En todo caso, el tipo de rotura dominante es transgranular.
También se han estudiado los efectos de distintas mezclas etanol-gasolina en diferentes aceros API 5LX con uniones soldadas ${ }^{[64]}$ y se ha comprobado que no se puede hacer una generalización de los resultados de los ensayos de corrosión bajo tensión cuando se emplean ensayos de deformación a baja velocidad, Strain Slow Rate Testing (SSRT), obtenidos en el laboratorio, respecto al comportamiento que pudieran presentar en servicio. En este mismo trabajo, se comprobó que las uniones soldadas no aumentaban su susceptiblidad a la CBT.

En la tabla IV, se recoge un resumen comparativo de los trabajos realizados por Shridar et al. ${ }^{[31]}$, Lou et al. ${ }^{[62]}$ y Beavers et al. ${ }^{[64]}$. Las medidas propuestas actualmente para minimizar este problema incluyen el empleo de inhibidores de la corrosión ${ }^{[23]}$, de tratamientos de alivio de tensiones, y la elección de aceros de elevada resistencia a corrosión localizada en presencia de estados tensionales en ambientes clorurados, como son los aceros inoxidables dúplex UNS $32101^{[65]}$

\section{CONCLUSIONES Y PERSPECTIVAS FUTURAS}

- En esta revisión se han descrito los estudios realizados sobre la corrosión que experimentan distintos sistemas metálicos, cuando entran en contacto con etanol y sus mezclas con gasolina. Debido a la influencia de numerosas variables experimentales involucradas en este tipo de investigaciones, tales como tipos y calidad del etanol utilizado, diferentes sistemas metálicos, condiciones físicas, tipo de ensayo, etc., es difícil comparar resultados y obtener conclusiones claras. En consecuencia, se recomienda la realización de trabajos experimentales, informar sobre la composición físico-química del etanol utilizado, incluyendo la posible adición de aditivos anticorrosión y desnaturalizantes y especificar al máximo las condiciones experimentales de los ensayos de corrosión electroquímica, en cuanto a una posible corrección de la caída óhmica, adición de sales para aumentar la conductividad, etc. A pesar de estas dificultades, a continuación se presentan una serie de conclusiones tras la revisión bibliográfica realizada:

- El etanol de elevado nivel de pureza no es un medio corrosivo, pero contiene algunas impurezas y especies químicas, fundamentalmente agua y cloruros, que condicionan su $\mathrm{pH}$, y que pueden provocar corrosión en las aleaciones utilizadas industrialmente.

- No se ha establecido una relación directa entre el aumento del contenido de etanol y sus mezclas con gasolina, con la corrosividad de estos combustibles. Para ello, sería necesario realizar ensayos con mezclas con mayor proporción de etanol. 
Tabla IV. Resumen de los estudios realizados por Sridhar et al. [31], Lou et al. ${ }^{[62]}$ y Beavers et al. ${ }^{[64]}$ de la susceptibilidad del fenómeno de corrosión bajo tensión en etanol y en distintos materiales

Table IV. Summary of the studies realized by Sridhar et al.. ${ }^{[31]}$, Lou et al..$^{[62]}$ and Beavers et al. ${ }^{[64]}$ in relation to stress corrosion cracking susceptibility of different materials produced by the ethanol

\begin{tabular}{|c|c|c|c|c|c|}
\hline Autores & $\begin{array}{l}\text { Materiales } \\
\text { ensayados }\end{array}$ & $\begin{array}{l}\text { Ensayos } \\
\text { realizados: } \\
\text { Ensayos de } \\
\text { deformación a } \\
\text { baja velocidad } \\
\text { (SSRT) }\end{array}$ & $\begin{array}{l}\text { Factores que } \\
\text { favorecen la } \\
\text { susceptibilidad } \\
\text { a la CBT }\end{array}$ & $\begin{array}{l}\text { Factores que } \\
\text { no influyen o } \\
\text { inhiben la } \\
\text { susceptibilidad } \\
\text { a la CBT }\end{array}$ & $\begin{array}{l}\text { Tipo de fractura } \\
\text { dominante }\end{array}$ \\
\hline $\begin{array}{l}\text { Sridhar } \\
\text { et al. }{ }^{[31]}\end{array}$ & $\begin{array}{l}\text { Acero al } \\
\text { carbono } \\
\text { ASTM A36 }\end{array}$ & $\begin{array}{l}\text { Velocidad de } \\
\text { deformación = } \\
4 \times 10^{-7} \mathrm{~s}^{-1}\end{array}$ & $\begin{array}{l}\text { - Oxígeno } \\
\text { disuelto. } \\
\text { - Contenido en } \\
\text { cloruros. } \\
\text { - Ácido acético } \\
\text { (0-56 mg/L). } \\
\text { - Inhibidor de la } \\
\text { corrosión } \\
(0,08 \mathrm{mg} / \mathrm{L}) \text {. }\end{array}$ & $\begin{array}{l}\text { - Contenido } \\
\text { en agua. } \\
\text { - Metanol } \\
(0-0,5 \%)\end{array}$ & $\begin{array}{l}\text { - Intergranular } \\
\text { con bajos } \\
\text { contenidos } \\
\text { decloruros } \\
\text { (<1 ppm). } \\
\text { - Transgranular } \\
\text { con altos } \\
\text { contenidos de } \\
\text { cloruros } \\
\text { ( } \geq 35 \mathrm{ppm}) .\end{array}$ \\
\hline $\begin{array}{l}\text { Lou } \\
\text { et al. }{ }^{[62]}\end{array}$ & $\begin{array}{l}\text { Acero al } \\
\text { carbono } \\
x 65\end{array}$ & $\begin{array}{l}\text { Velocidad de } \\
\text { deformación }= \\
1 \times 10^{-7} \mathrm{~s}^{-1} \mathrm{a} \\
2 \times 10^{-6} \mathrm{~s}^{-1}\end{array}$ & $\begin{array}{l}\text { - Contenido en } \\
\text { cloruros. } \\
\text { - Contenido en } \\
\text { agua }(2,5 \%) \text {. } \\
\text { - Oxígeno } \\
\text { disuelto. }\end{array}$ & $\begin{array}{l}\text { - Contenido } \\
\text { en agua } \\
\text { (5\%). } \\
\text { - Valores altos } \\
\text { de pHe. }\end{array}$ & - Transgranular. \\
\hline $\begin{array}{l}\text { Beavers } \\
\text { et al. }{ }^{[64]}\end{array}$ & $\begin{array}{l}\text { Aceros API } \\
5 \mathrm{LX} \text { con } \\
\text { uniones } \\
\text { soldadas }\end{array}$ & $\begin{array}{l}\text { Velocidad de } \\
\text { deformación }= \\
9,53^{-6} \mathrm{~s}^{-1}\end{array}$ & $\begin{array}{l}\text { - Oxígeno } \\
\text { disuelto. } \\
\text { - Procedimiento } \\
\text { de soldadura. }\end{array}$ & $\begin{array}{l}\text { - Modo de } \\
\text { Fractura } \\
\text { mixto. }\end{array}$ & \\
\hline
\end{tabular}

- Se puede afirmar que todos los materiales metálicos, objeto de estudio a lo largo de los años, excepto las aleaciones Al-Zn, Zamak, presentan bajas o nulas velocidades de corrosión en contacto con los combustibles E5 y E10 que emplean el etanol deshidratado con inhibidores de corrosión. Para porcentajes de etanol hidratado de 20/25\%, se hace necesaria la utilización de recubrimientos protectores, especialmente de cadmio y cromo.

- Se ha demostrado la posibilidad de la existencia del fenómeno de corrosión bajo tensión en componentes metálicos empleados en las plantas de obtención, distribución y almacenaje de etanol. El factor que ejerce mayor influencia es el oxígeno disuelto, y el tipo de fractura principal observado es transgranular. Por último, se desea resaltar la necesidad de realizar más investigación para ayudar al desarrollo y fomento de la industria que emplea etanol y sus mezclas con gasolina, y conocer la susceptibilidad a la corrosión de materiales metálicos en presencia de determinadas mezclas de gasolina-etanol.

\section{REFERENCIAS}

[1] L. Cummins, Internal Fire, Society of Automotive Engineers (Eds.), 1989, p.p. 81.

[2] M. Lapuerta, O. Armas, J. Rodríguez-Fernández, Prog. Energy Combust. Sci. 34 (2008) 198-223.

[3] C. D. Rakopoulus, K. A. Antonopoulos, D. C. Rakopoulos, D. T. Hountalas y E. G. Giakoumis, Energ. Convers. Manage, 47 (2006) 3.272-3.287.

[4] G. Labeckas y S. Slavinskas, Energ. Convers. Manage, 47 (2006) 3.272-3.287. 
[5] European Parliament, Directive 2003/30/EG: "On the promotion of the use of biofuels or other renewable fuels for transport", 2003.

[6] A. Demirbas, Energ. Sources A 27 (2005) 327-337.

[7] R. K. Niven, Renew. Sust. Energ. Rev 9 (2005) 535-555.

[8] N. L. Singh, P. Srivastava y P.K. Mishra, J. Sci. In. Res. 68 (2009) 617-623.

[9] V. Codling, Proc. Internat. Symp. on Alcohol Fuel Technology, Inst de Pesquisas Tecnologicas do Estado de Sao Paulo (Eds.), Guaruja, Brasil, 1980, pp. 285-288.

[10] ASTM D 4806-03: "Standard Specification for Denatured Fuel ethanol for Blending with Gasolines for Use as Automotive Spark-Ignition Engine Fuel”, Annual Book of ASTM Standards, ASTM International, 2003.

[11] Brazilian National Petroleum Agency (ANP): "Brazilian anhydrous \& Hydrated ethanol specifications,” 2002.

[12] prEN 15376: "Draft standard for non-denatured automotive ethanol as a blending component in gasoline at up to and including $5 \%$ " European Committee for Standardization (CEN), 2007.

[13] Renewable Fuels Association: "Fuel Ethanol, Industry Guidelines, Specifications and Procedures", EE.UU., 2005.

[14] F. Mansfield, J. Chem. Soc. 120 (1973) 188-192.

[15] C. A. Farina, G. Faita y F. Olivani, Corros. Sci. 18 (1978) 465-479.

[16] S. Sternberg y V. Branzôi, Electrochem. Acta 29 (1984) 15-19.

[17] Y. Roques, G. Mankowski, G. Chatainer y F. Dabosi, Corrosion 40 (1984) 561-567.

[18] D. D. N. Singh y M. K. Banerjee, Corrosion 42 (1986) 156-161.

[19] P. L. De Anna, Corros. Sci. 25 (1985) 43-53.

[20] J. Banas, Electrochem. Acta 32 (1987) 871-875.

[21] V. B. Singh y V. K. Singh, Mater. Trans. 32 (1991) 251-256.

[22] E. Heitz, Corrosion of Metals in Organic Solvents, Plenum Publishers Eds., 1974, pp. 149-243.

[23] F. Gui y N. Sridhar, Corrosion 66 (2010) 045-050.

[24] K. Nakajima y Y. Yahagi, J. Lubr. Technol. 105 (1983) 552-558.

[25] V. P. Persiantseva, I. L. Rozenfeld, V. E. Zorina, E. K. Enikeev y M. I. Churaeva, Prot. Met. 15 (1979) 245-248.

[26] V. V. Ekelik, V. P. Grigoriev y G. N. Ekilik, Zashch. Met. 9 (1973) 50-51.

[27] X. Nie, X. Li y D. Northwood, Mater. Sci. Forum 546-549 (2007) 1.093-1.100.

[28] D. Korotney, Water Phase Separation in Oxygenated Gasoline, Fuel Studies and Standards Branch, EEUU, 1995.
[29] JAMA Position Statement FQ-01 "Quality of Bioethanol and use of ethanol-blended Gasoline”, 2009, pp. 1-3.

[30] M. Kabasakaloglu, I. Kalyoncu y T. Kiyak, Appl. Surf. Sci. 135 (1998) 188-192.

[31] N. Sridhar, K. Price, J. Buckingham y J. Dante, Corrosion 62 (2006), 687-702.

[32] S. M. Traldi, I. Costa y J. L. Rossi, Rev. Metal. Madrid, Vol. Extr., (2003) 86-90.

[33] H. Jahnke y M. Schoenborn, Mater. Corr. 36 (1985) 561-566.

[34] R. S. Goncalves, N. M Coradini y W. X. Olivera, Corr. Sci. 33 (1992) 1.667-1.675.

[35] D. K. Tanaka y S. Wolynec, Proc. 5th. International Fuel Technology [s.n.], Auckland, Nueva Zelanda, 1982, pp. 461-466.

[36] E. Cavalcanti, V. G. Wanderley, T. R. V. Miranda y L. Uller, Electrochim. Acta, 32 (1987) 935-937.

[37] V. B. Singh y V.K. Singh, Mater. Trans. 32 (1991) 756-762.

[38] I. Sekine, M. Yuasa y K. Kohara, Corr. Sci. 31 (1990) 579-584.

[39] R. R. Wiggle, V. Hospadaruk, E. A. Styloglou, G. K. Chui y W. D.Tallent, Proc. Internat. Symp. on Alcohol Fuels Technology, Inst de Pesquisas Tecnologicas do Estado de Sao Paulo (Eds.), Sao Paulo, Brasil, 1980, pp. 441-449.

[40] S. Wolynec y D. K. Tanaka, Proc 9 Seminário Nacional de Corrosão - SENACOR, RJ. Anais. Rio de Janeiro, Brasil, ABRACO (Eds.), 1982. pp. 166-176.

[41] S. Wolynec y D. K. Tanaka, Proc 3 Simpósio Nacional de Corrosão na Produção e Utilização do Álcool, ABRACO (Eds.), Rio de Janeiro, Brasil, 1983, pp. 1-38.

[42] D. K. Tanaka, Z. P. Kajimoto y S Wolynec, Proc 3o Simpósio Nacional de Corrosão na Produção e Utilização do Álcool, ABRACO (Eds.), Rio de Janeiro, Brasil, 1983, pp. 94-103.

[43] Z. P. Kajimoto y S. Wolynec, Proc 3o Simpósio Nacional de Corrosão na Produção e Utilização do Álcool, ABRACO (Eds.), Rio de Janeiro, Brasil, 1983, pp. 237-249.

[44] S. Wolynec y D. K. Tanaka, Proc. Encontro Brasileiro de Tratamento de Superfície - EBRATS'83, ABTS (Eds.), São Paulo, Brasil, 1983, pp. 263-283.

[45] Z. P. Kajimoto, S. Wolynec y D. K. Tanaka, Proc. 6 th. Proc. Internat. Symp. on Alcohol Fuel Technology [s.n.], Ottawa, Canadá, 1984, pp. 1.225-1.231.

[46] S. Wolynec y D. K. Tanaka, Proc. 9th. Inter Congress on Metallic Corrosion, National Research Council (Eds.), Toronto, Canadá, 1984, pp. 468-474.

[47] Orbital Engine Company, Report to the Department of the Environment and Heritage to Environment, Australia, December 2002. 
[48] Orbital Engine Company: A Literature Review Based Assessment on the Impacts of a $10 \%$ and $20 \%$ Ethanol Gasoline Fuel Blend on Non-Automotive Engines, Australia, 2002.

[49] Orbital Engine Company, Market Barriers to the Uptake of Biofuels Study: A Testing based assessment to determine impacts of a $20 \%$ ethanol gasoline fuel blend on the Australian passenger vehicle fleet-Ethanol- 2000 hrs Material Compatibility Testing, Australia, 2002.

[50] Orbital Engine Company, Market Barriers to the Uptake of Biofuels Study: A Testing Based Assessment to Determine Impacts of a $10 \%$ and 20\% Ethanol Gasoline Fuel Blend on Non-Automotive Engines- 2000 hrs Material Compatibility Testing, Australia, 2003.

[51] B. Jones, G. Mead, P. Steevens y M. Timanus, The Effects of E20 on Metals Used in Automotive Fuel System Components, Minnesota Center for Automotive Research at Minnesota State University, Mankato, Minnesota, EEUU, 2008.

[52] Metals Handbook, Vol. 13, Corrosion, ASM International, 1986, pp. 1.180-1.182.

[53] L. Lunde y R. Nyborg, Proc. Corrosion/1989, NACE International Eds., 1989, pp. 1-20.

[54] S. B. Farina, G. S. Duffo y J. R. Galvele, Corrosion 59 (2002) 436-442.
[55] D. J. Simbi y J. C. Scully, Corr. Sci. 34 (1993) 1.743-1.750.

[56] D. J. Simbi y J. C. Scully, Corrosion 53 (1997), 298-305.

[57] Metals Handbook, Vol. 13, Corrosion, Chapter on Corrosion Titanium an Titanium Alloys, ASM International, 1987 Ed., pp. 687-688.

[58] E. Sergent y Y. Roques, Corros. Sci. 36 (1994) 653-667.

[59] Y. Roques, G. Mankowski, G. Chatainier y F. Dabosi, Electrochem. Acta 32 (1987) 971-976.

[60] R. D. Kane y J. G. Maldonado, Technical Report 939-D, American Petroleum Institute (API), $1^{\text {a }}$ Edición, Washington, EE.UU, 2003.

[61] R. D. Kane y J. G. Maldonado, Technical Report 939-D, American Petroleum Institute (API), $2^{\underline{a}}$ Edición, Washington, EE.UU, 2007.

[62] X. Lou, D. Yang y P. M. Singh, Corrosion 65 (2009) 785-797.

[63] X. Lou, D. Yang y P. M. Singh, J. Electrochem. Soc., 157 (2010) C86-C94.

[64] J. Beavers, N. Sridhar, C. Zamarin, Corrosion 2009, Annual Conference and Exhibition, Ed. NACE Int, paper n. 09532, Atlanta, EE.UU, pp. 1-16.

[65] E. Torsner, Corros. Eng. Sci. Tech. 45 (2010) $42-48$. 